\title{
Surface wave focusing and acoustic communications in the surf zone
}

\author{
James C. Preisig \\ Department of Applied Ocean Physics and Engineering, Woods Hole Oceanographic Institution, \\ Woods Hole, Massachusetts 02543 \\ Grant B. Deane \\ Marine Physical Laboratory, Scripps Institution of Oceanography, La Jolla, California 92093
}

(Received 22 September 2003; revised 17 May 2004; accepted 18 May 2004)

\begin{abstract}
The forward scattering of acoustic signals off of shoaling surface gravity waves in the surf zone results in a time-varying channel impulse response that is characterized by intense, rapidly fluctuating arrivals. In some cases, the acoustic focusing by the curvature of the wave crest results in the formation of caustics at or near a receiver location. This focusing and the resulting caustics present challenges to the reliable operation of phase coherent underwater acoustic communications systems that must implicitly or explicitly track the fluctuations in the impulse response. The propagation physics leading to focusing are studied with both experimental data and a propagation model using surface wave profiles measured during the collection of the experimental data. The deterministic experimental and modeled data show good agreement and demonstrate the stages of the focusing event and the impact of the high intensity arrivals and rapid fluctuations on the ability of an algorithm to accurately estimate the impulse response. The statistical characterization of experimental data shows that the focusing by surface gravity waves results in focused surface reflected arrivals whose intensity often exceeds that of the direct arrival and the focusing and caustic formation adversely impacts the performance of an impulse response estimation algorithm. (C) 2004 Acoustical Society of America. [DOI: 10.1121/1.1771591]
\end{abstract}

PACS numbers: 43.30.Re, 43.60.Cg, 43.60.Dh [EJS]

Pages: $2067-2080$

\section{INTRODUCTION}

The surf zone presents a challenging physical environment for underwater acoustic communications systems. This range dependent and highly reverberant environment is complicated by acoustic scattering from the shoaling gravity wave field and attenuation of acoustic signals by dense plumes of bubbles. The surface wave field can cause significant fluctuations in the delay of surface scattered signals, the breaking surf injects dense plumes of bubbles in the water column resulting in highly variable scattering and attenuation of propagating signals, and wave front focusing of surface reflected signals results in the formation of transient caustics. Prior work has examined characteristics of sonar performance in the surf zone environment ${ }^{1}$ and the influence of bubble clouds on acoustic propagation. ${ }^{2}$ While all of the above listed acoustic effects impact the performance of acoustic communications systems, this paper will analyze the characteristics of surface wave focusing and transient caustics and their potential impact on phase coherent underwater acoustic communications systems.

Wave front focusing caused by acoustic reflections from shoaling surface waves is a significant propagation phenomena in the near-shore environment (see, for example, Ref. 3 for a treatment of caustics and deterministic surface scattering). As will be shown in this paper, the caustics resulting from this focusing often have amplitudes much greater than those of the direct arrival, occur at delays much greater than those of the direct arrival, are highly transient in nature often appearing and disappearing within a few hundred milliseconds, and may exhibit abrupt phase shifts.

In the surf zone environment where the time-varying channel impulse response has significant multipath-induced delay spread, phase coherent signal demodulation relies implicitly or explicitly on accurate estimation of this impulse response. It will be shown later in this paper that the characteristics of the surface wave focusing and the resulting transient caustics described in the previous paragraph pose challenges to the reliable estimation of the channel impulse response and therefore the reliable operation of such signal demodulation algorithms.

To illustrate the importance of acoustic wave front focusing by surface gravity waves, consider Fig. 1. This figure shows the mean and maximum magnitude squared (intensity) of the channel impulse response measured $40 \mathrm{~m}$ from a source in the surf zone as a function of relative delay. These sample statistics were calculated using observations over a 9 s time period as a surface wave passed overhead. The details of the processing to generate these statistics are contained in Sec. IV and the Appendix. The direct and bottom arrivals are relatively stable and show little difference between their mean and maximum values. However, the surface reflected arrivals are highly dynamic. While their mean values are significantly below those of the direct and bottom arrivals, their peak values exceed those of the direct and bottom arrivals by as much as $7 \mathrm{~dB}$. At first glance, these increased magnitudes are surprising since surface scattering and geometrical spreading would lead one to expect the kind of magnitude decay observed in the mean values. However, the 


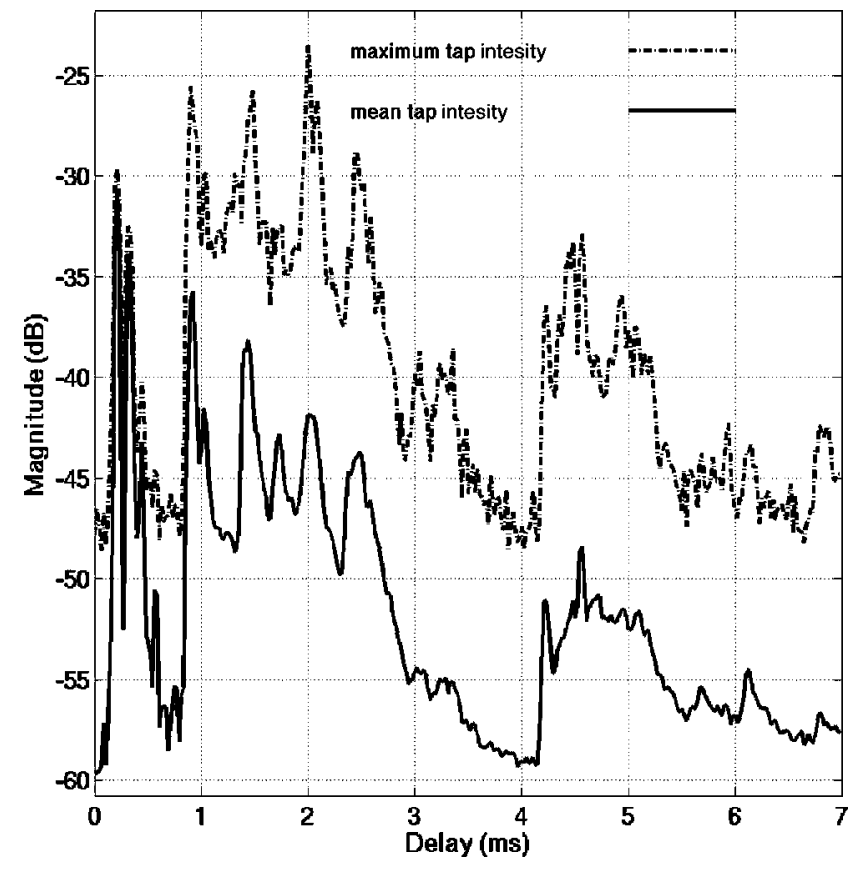

FIG. 1. Maximum and mean intensity of estimated time-varying channel impulse response. The first and second signficant arrivals, both occurring before $0.5 \mathrm{~ms}$ in delay, are the direct and bottom bounce arrivals, respectively. The next four significant arrivals occurring in the interval from approximately 0.9 to $2.5 \mathrm{~ms}$ in delay represent the surface bounce, surfacebottom bounce, bottom-surface bounce, and bottom-surface-bottom bounce paths, respectively. The arrivals occurring after $2.5 \mathrm{~ms}$ in delay all represent paths with more than one surface bounce.

analysis in this paper will show that acoustic focusing by surface gravity waves can lead to surface scattered arrivals that are significantly greater in amplitude than direct and bottom scattered paths. As noted earlier, these high amplitude arrivals are highly transient in nature and pose challenges to acoustic communications systems in the surf zone.

This paper combines results from theoretical analysis, analysis of data generated using an acoustic propagation model, and the analysis of experimental data. A description of the propagation experiment labeled Wavefronts II from which data was obtained can be found in Sec. II. Section III begins with a description of the channel impulse response both as measured during the Wavefronts II experiment and modeled using the Wavefronts acoustic propagation model. ${ }^{4}$ It then goes on to describe the anatomy of an acoustic focusing event and concludes with an analysis of the impact of the acoustic focusing on the estimation of the channel impulse response and the performance of phase coherent acoustic communications systems. Section IV presents statistical characterizations of the channel impulse response and performance of the least-squares algorithm used to estimate it. Section V summarizes the contributions of the paper. The Appendix details the processing methods used to estimate the channel impulse response and scattering functions observed during the experiment.

The impulse response intensities, scattering function intensities, received signal levels, and signal estimation residual error (SER) levels presented in the paper are all represented in $\mathrm{dB}$. Unless otherwise noted, the reference for these is the estimated intensity of the direct arrival of the

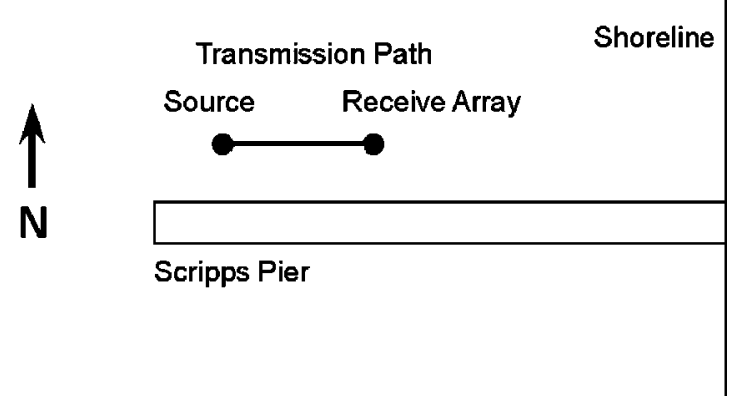

FIG. 2. Wavefronts II experiment top view.

channel impulse response one meter from the source transducer during the Wavefronts II experiment.

\section{THE WAVEFRONTS II EXPERIMENT}

The Wavefronts II transmission experiment took place in the surf zone in approximately $6 \mathrm{~m}$ deep water, $30 \mathrm{~m}$ north of Scripps Pier in December 2001. Top and side views of the experiment geometry are shown in Figs. 2 and 3. Broadband signals with center frequencies ranging from 12 to $26 \mathrm{kHz}$ were generated with an ITC 1007 source jetted $2.0 \mathrm{~m}$ above the seafloor. The signals were transmitted $38 \mathrm{~m}$ inshore to an array of 3, ITC $6050 \mathrm{C}$ hydrophones vertically spaced at 0.5 $\mathrm{m}$ intervals, with the bottom hydrophone $1.51 \mathrm{~m}$ above the seafloor. A reference hydrophone was deployed at the same depth as and $0.71 \mathrm{~m}$ shoreward of the source and was used to monitor the source signal and level. The experiment geometry was designed to allow surface reflected arrivals to be time-separated from other paths with the available source bandwidth and this was achieved.

An array of 8 pressure sensors was deployed just above the seafloor along the acoustic propagation path to allow shoaling surface gravity waves to be monitored simultaneously with the acoustic transmissions. The sensors of the pressure array were sampled at a rate of $5 \mathrm{~Hz}$. The water depth and pressure sensor locations were surveyed by divers during the experiment, and are identified in Fig. 3. The solid line shows a least-squared fit to the surveyed depths. The linear regression analysis shows that the seafloor had an almost constant slope of $2.0^{\circ}$ along the propagation path. The sea surface corresponds to an actual surface gravity wave profile measured during the time of the transmissions, and illustrates the fact that generally only one wave crest was found between the source and the receive array. This is an important point that we will return to later.

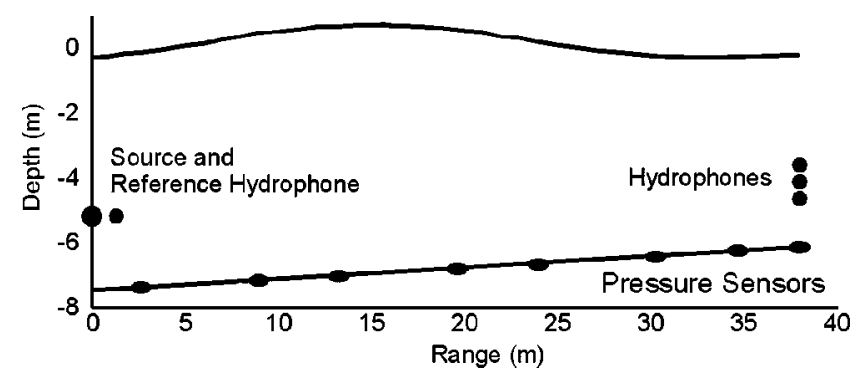

FIG. 3. Wavefronts II experiment side view. 


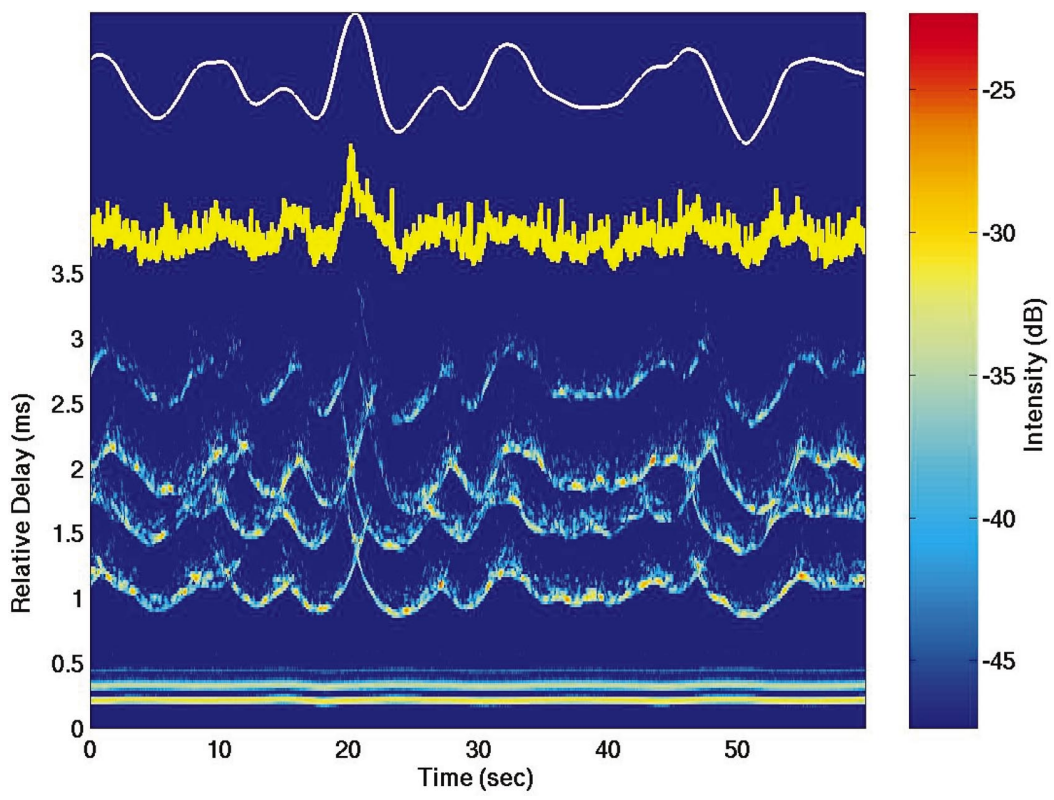

FIG. 4. Surface wave height, SER, and intensity of estimated time-varying channel impulse response. The horizontal lines at the bottom represent the overlapping direct arrival and first bottom bounce. The time-varying arrivals, in order from bottom to top, are the first surface bounce, the surface-bottom bounce, the bottom-surface bounce, and the bottom-surface-bottom bounce. The top white line shows the measured surface wave height near the specular reflection point of the first surface scattered path. The trough to peak excursion on this plot is $1.21 \mathrm{~m}$. The yellow line below the surface wave height is a plot of the magnitude of signal estimation residual error (SER) realized by the algorithm used to estimate the channel impulse response. This plot is in $\mathrm{dB}$ and the minimum to maximum error excursion is $10.74 \mathrm{~dB}$.

The sound speed during the experiment was measured to be $1503 \mathrm{~m} / \mathrm{s}$. The sound speed, absorption, and density of the seafloor can be estimated using Buckingham's geoacoustic theory for sediments provided the mean grain size of the sand is known. ${ }^{5}$ The mean diameter of grains in a sample of sand collected from La Jolla Shores beach was analyzed photographically and estimated to be 573 microns. The geoacoustic parameters corresponding to this diameter and taken to be representative of the seafloor are a density of 2048 $\mathrm{kg} / \mathrm{m}^{3}$, a sound speed of $1757 \mathrm{~m} / \mathrm{s}$ (corresponding to a critical angle in the seafloor of $31.2^{\circ}$ ) and an absorption of $0.85 \mathrm{~dB}$ per wavelength.

The transmit and receive signals were sampled at a rate of $96 \mathrm{kHz}$. Transmit signals were generated with center frequencies of 12,18 , and $26 \mathrm{kHz}$, and were prefiltered to provide an approximately flat system frequency response over a bandwidth of the inverse of the pulse or symbol duration around the center frequency of the signal. Signals were transmitted continuously for approximately $60 \mathrm{~s}$ with a several second pause between each transmission period. Thirty such $60 \mathrm{~s}$ transmissions were made with each signal. Three signal formats were transmitted. The first was a binary phase shift keyed (BPSK) signal modulated by continuous repetitions of a 4095 point maximum length shift register sequence (m-seq). ${ }^{6}$ The symbol rates for the 12,18 , and $26 \mathrm{kHz}$ signals were 16000,24000 , and 48000 symbols per second, respectively. The second transmitted signal consisted of a single cycle of a pulse at the 12,18 , or $26 \mathrm{kHz}$ center frequency with a repetition rate of 16 pulses per second. The final transmitted signal consisted of interleaved single cycle pulses and a BPSK signal modulated with 13 symbol Barker code. The data analyzed and presented in this paper is the $18 \mathrm{kHz}$ m-seq data.

\section{A SURFACE WAVE FOCUSING EVENT AND ITS IMPACT ON ACOUSTIC COMMUNICATIONS}

The intensity of the channel impulse response estimated from data collected during the Wavefronts II experiment, the surface wave height at the time of data collection, and the signal estimation residual error (SER) realized by the algorithm (see Appendix) used to estimate the channel impulse response are shown in Fig. 4. While the delay spread of the impulse response is on the order of $7 \mathrm{~ms}$, this figure shows the arrivals for only the first $3.5 \mathrm{~ms}$ of the arrival structure. This includes the direct and first bottom bounce arrivals as well as all of the arrivals with a single surface bounce. A low SER indicates that the algorithm is providing a good estimate of the channel impulse response.

The variations in arrival time and intensity of the surface scattered paths are clearly associated with the passage of surface gravity waves over the experiment transmission path. Transient caustics are formed by the wave front focusing property of shoaling surf. As open ocean swell approaches the coastline and begins to interact with the sea floor, it refracts so that wave fronts become parallel to the isobaths and it grows in amplitude. As it shoals, each wave crest forms an acoustic mirror with its own characteristic shape and focusing properties. The surf therefore forms a series of timevarying mirrors that create moving focal regions of scattered sound.

Several important features can be observed in the data. The first is that the passage of a peak and trough results in an asymmetric pattern of arrival time fluctuations and, most significantly, signal estimation errors. The passage of a trough results in a single arrival that first decreases and then increases in delay so the range rate of the arrival is approxi- 


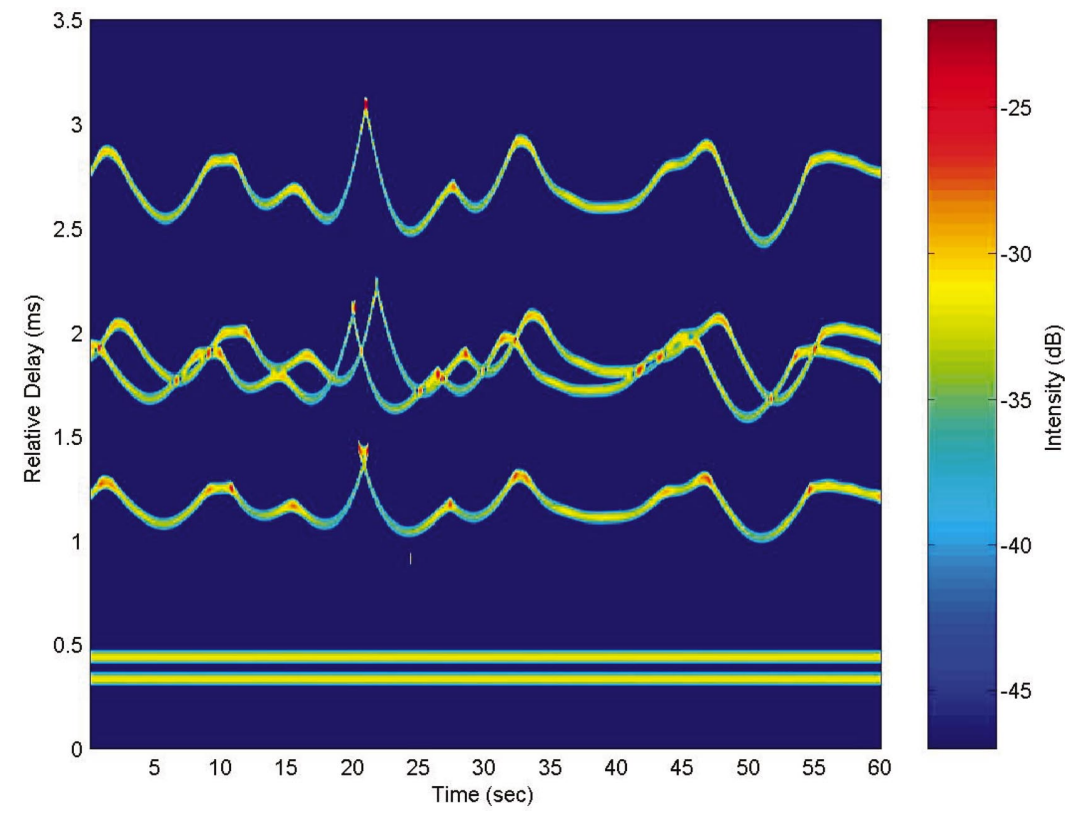

FIG. 5. Intensity of modeled time-varying channel impulse response.

mately zero. The term "range rate" is used herein to mean the apparent rate at which the location of an arrival is increasing or decreasing in delay. In contrast, the passage of a peak often results in two arrivals, one increasing and the other decreasing in delay, crossing with high range rates. The high range rates result in a large signal estimation error. This is most noticeable with the large wave crest that passed over the specular point at a time of $20 \mathrm{~s}$ in the plot. This pattern will be discussed in detail in Secs. III B and III C.

The second feature is that the intensity of the received signal at the caustics is larger than the direct arrivals, despite the fact that they have travelled along a longer path and been scattered by small-scale surface roughness. The caustics can appear at a delay that is significant (from a communications context) with respect to the direct arrival. In addition, the caustics can appear and disappear in the span of a few hundred milliseconds.

In Sec. III A which follows, the agreement of the estimated impulse response and the impulse response predicted with the Wavefronts acoustic propagation model is illustrated and limitations are discussed. In Sec. III B, the model results are used to illustrate the formation of a transient caustic and its relevant features during the passage of a single wave peak observed during the Wavefronts II experiment. This section is concluded with Sec. III C which uses acoustic data from the same wave peak event to further illustrate the relevant features of the transient caustic and their impact on the performance of a least squares channel estimation algorithm.

\section{A. The Wavefronts acoustic propagation model and comparison with experimental data}

A new method of modeling underwater sound propagation, called Wavefront Modeling, has been developed by Tindle. ${ }^{4}$ The method is based on a Hankel transformgeneralized Wentzel-Kramers-Brillouin (WKB) solution of the wave equation. The resulting integral leads to a new form of ray theory which is valid at relatively low frequencies and allows evaluation of the acoustic field on both the illuminated and shadow sides of caustics and at cusps where two caustics meet to form a focus. The method is much faster than other standard methods, is able to handle rapid range dependence, and the phase, amplitude, and travel time of broadband acoustic pulses are obtainable directly from a simple graph of ray travel time as a function of depth at a given range. The model can handle the following features of the environment or acoustic field: real but smooth surface waves from experimental data, range dependent smooth bathymetric variations, and cusps and caustics. The model can propagate real waveforms enabling direct comparison of modeled received waveforms and waveforms received during field experiments.

The Wavefronts model calculated the first $3.5 \mathrm{~ms}$ of the impulse response arrival structure shown in Fig. 5. The surface wave field used as an input to the model for this $60 \mathrm{~s}$ period was the same as that measured during the time when the acoustic signals used in generating Fig. 4 were gathered. The model shows good qualitative agreement with the impulse response estimates generated from the experimental data. The model results show the high intensity of the surface scattered arrivals as each wave peak passes and a lack of high intensity arrivals during the passage of wave troughs.

Of equal importance are the differences between the experimental data and model results. There is fine scale structure apparent in the data that is not seen in the model results. It is believed that this structure is due to the presence of small scale surface roughness that could not be detected by the bottom-mounted pressure sensors used during the experiment. This surface roughness is therefore not reflected in the model results. There are also limitations inherent in the least squares algorithm used to estimate the channel impulse response from the experimental data (see Appendix). The primary limitation is the $25 \mathrm{~ms}$ averaging time used by the 


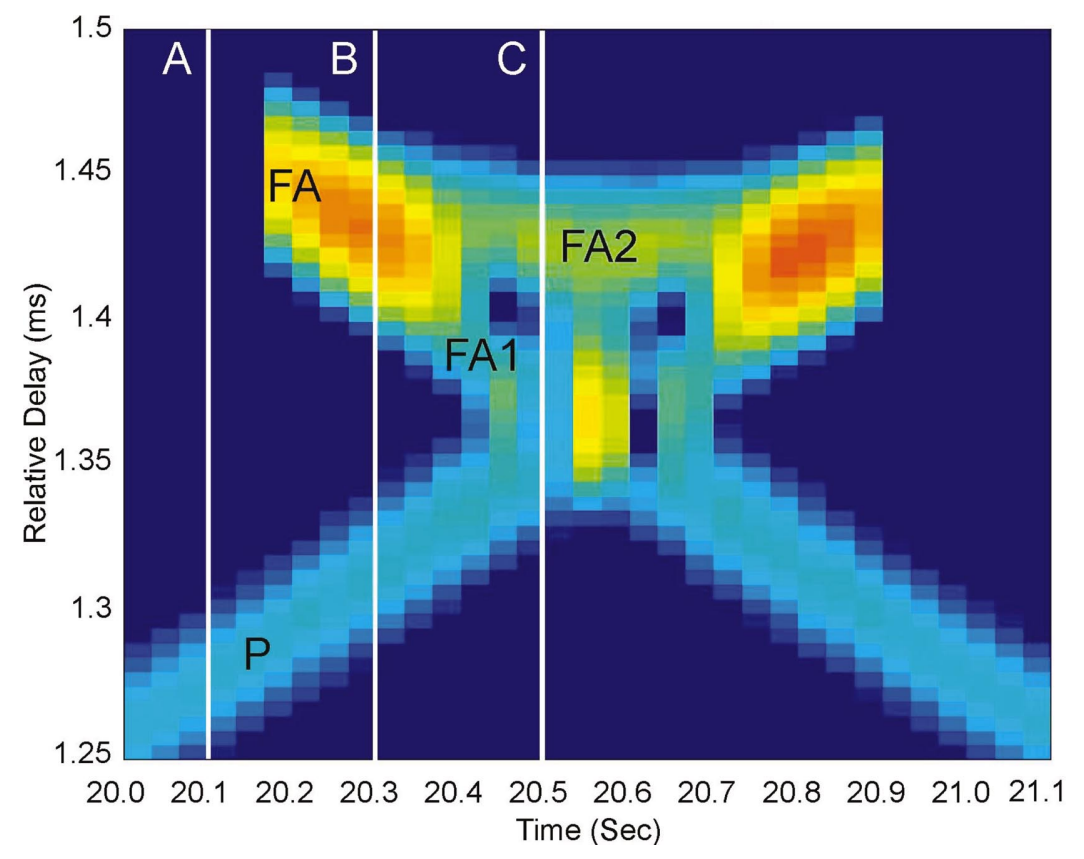

FIG. 6. Intensity of modeled time-varying channel impulse response during focusing event. The times labeled A, B, and C correspond to the three times for which the angle/depth diagrams are shown in Fig. 9. The arrival labels (P, FA, FA1, and FA2) correspond to the similarly labeled arrivals in Figs. 7, 9, and 10. Time A corresponds to the shadow zone of the focused arrival FA. Time B is just after the formation of the caustic. At time C, the saddle points for the focused arrival have diverged and the arrival has split into two arrivals labeled FA1 and FA2.

algorithm which can both smear estimates of arrivals in time and highly attenuate arrivals appearing at Doppler shifts of greater than about $20 \mathrm{~Hz}$.

The same model and experiment results focusing on the first surface bounce arrival in the vicinity of the time of $20 \mathrm{~s}$ are shown in Figs. 6 and 7, respectively. Noting the slight offset of the plots in the delay variable, the model results are seen to capture the essential features of the caustic seen in the experimental data. Notwithstanding the fine scale structure present in the data and absent from the model results, the model does provides a sufficiently detailed reproduction of the data to warrant its use as an analysis tool.

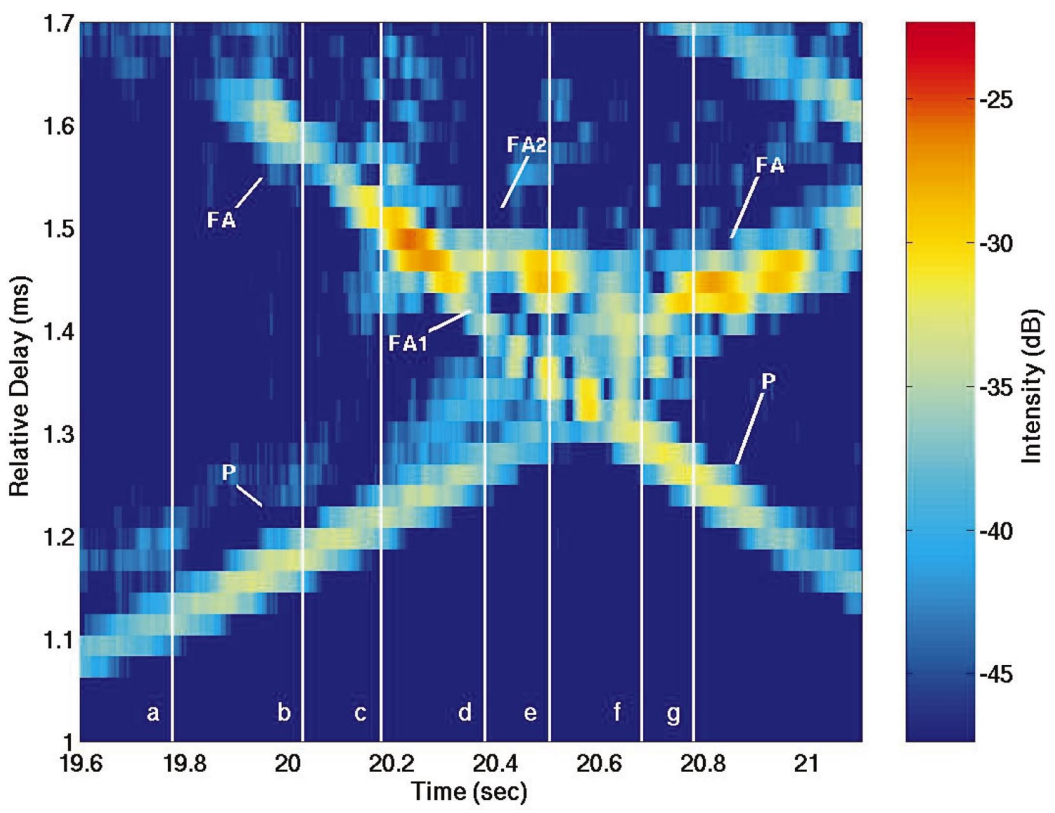

FIG. 7. Intensity of estimated time-varying channel impulse response during focusing event. The vertical white lines, labeled a-g mark significant points of time in the evolution of the surface scattered arrival. The arrival labels (P, FA, FA1, and FA2) correspond to the similarly labeled arrivals in Figs. 6, 9, and 10. The single ray arrival is the retreating primary arrival is labeled P. At time b, the advancing folded wave front arrival, labeled FA has appeared. The arrival has not yet formed a caustic. At time $\mathrm{c}$ the caustic is forming as the folded arrival gains in intensity. At time d the caustic has passed, the saddle points are diverging, and the folded wave front arrival has split into two arrivals labeled FA1 and FA2. The later of the two arrivals (FA2) is scattering off of the peak of the wave crest and is therefore stationary in delay and exhibits no Doppler shift. The evolution of the wave front focusing continues through times e-g. FA1 becomes the primary arrival and is advancing. FA2 and P eventually merge to form a caustic at time $g$ near the end of the focusing event. 


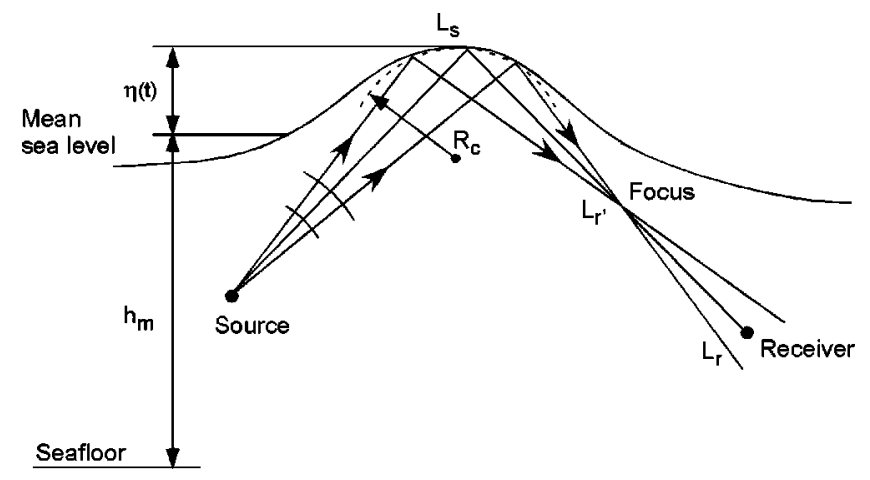

FIG. 8. Qualitative description of acoustic focusing by surface waves. The source to surface wave distance $\left(L_{s}\right)$ and the surface wave to focal point distance $\left(L_{r^{\prime}}\right)$ distance are related by the wave focal length $\left(L_{f}\right)$.

\section{B. The anatomy of a caustic}

This section begins with a qualitative description of sound focusing by shoaling gravity waves and then illustrates the underlying physical structure with model calculations for the wave that caused the intensification of the first surface bounce arrival about $20 \mathrm{~s}$ into Fig. 4. A schematic depiction of wave field focusing is shown in Fig. 8. The shoaling surface gravity wave has an instantaneous height $\eta(t, r)$ as a function of time, $t$ and range $r$, and the local radius of curvature of the wave crest at the specular reflection point is $R_{c}$. The mean water depth is $h_{m}$ and the total water depth is $h_{w}(t, r)=h_{m}+\eta(t, r)$. Sound emitted by the source, $L_{s}$ meters from the wave crest is shown focused $L_{r^{\prime}}$ meters from the wave crest, due to scattering by the (assumed locally spherical) surface. The relationship between $L_{s}$ and $L_{r^{\prime}}$ is determined by the wave focal length, $L_{f}$.

The focusing properties of the shoaling wave crests depends on the detailed shape of the air-water interface at the time of pulse reflection, which includes asymmetry in onshore direction (nonlinear effects result in the leading edge of the crest being steeper than the trailing edge) and scattering from features smaller than an acoustic wavelength. Based on measurements of shoaling wave crests taken from the pressure array deployed during the Wavefronts II experiment, it is assumed that to first order the shape of wave crest can be approximated as a cylindrical shell. As explained in Ref. 7, the normal-incidence focal length of a spherical section depends on the section radius of curvature and the relationship $L_{f}=R_{c} / 2$ is assumed for the focal length of the waves.

The Wavefronts model calculation of the impulse response intensity during the passage of the wave crest over the acoustic transmission path is shown in Fig. 6. The intensity versus delay and time clearly shows the "butterfly" pattern characteristic of the passage of a folded wave front past the receiver. The two intense spots on the wings are caustics, which occur on the boundary between insonified and acoustic shadow regions. Note that the passage of the wave crest does not result in a single arrival increasing and then decreasing in delay. Instead, the surface wave curvature and wave front folding results in two distinct arrivals, one increasing in delay and the other decreasing in delay, which merge and the separate as the wave crest passes through the specular region. As will be shown in Sec. III C, this structure in the wave field results in a significant Doppler spread of the channel scattering function during the focusing event. In the figures showing detailed arrival structure, different arrivals bear the labels, P, FA, FA1, and FA2. The label P indicates a primary surface scattered arrival not subject to strong surface wave focusing while the label FA (with or without a number) indicates a surface scattered arrival arising from a folded wave front which results in surface wave focusing.

The detailed arrival structure of the focused wave field has been analyzed at the three times, A, B, and C annotated in Fig. 6. The results are shown in Fig. 9. The three plots on the left show the depth versus launch angle of small segments of acoustic wave fronts at the receiver range, propagated through the water column and interacting with both the surface and bottom. The segment of wave front between launch angle $1^{\circ}$ to $-7^{\circ}$ corresponds to the direct-path arrival, and the segment between $-7^{\circ}$ and $-28^{\circ}$ corresponds to the surface-interacting wave fronts. The plots on the right show ray paths which pass through the receiver after reflection off the surface. The launch angle of these rays corresponds to the points in the left hand plots at which the depth versus launch angle curve intersects a line at the receiver depth. Those points on the surface-interacting segment of the curves have been annotated with a small circle. Points of intersection on other segments of the curve (e.g., the directpath segment, etc.) also correspond to arrivals at the receiver, but do not correspond to the portion of the channel impulse response considered here.

At time A in Fig. 9, there is only a single intersection point, corresponding to a single ray path connecting the source and the receiver. Accordingly, there is only a single arrival at the receiver at time A in Fig. 6. The region at time A between 1.4 and $1.5 \mathrm{~ms}$ delay is a shadow zone for the folded wave front that appears between times $\mathrm{A}$ and $\mathrm{B}$. At time $\mathrm{B}$, there are three points of intersection, two of which occur spaced closely together around $-20^{\circ}$. The two near-by saddle points form the caustic that first appears between A and $\mathrm{B}$, while the third point remains distinct. At time $\mathrm{C}$, all three points are distinct. If the geometry is such that the receiver lies right on the focal point, then all three saddle points merge. This pattern of arrivals is the underlying structure resulting in the advancing and retreating arrivals observed in the data in Figs. 4 and 7.

The following subsection will illustrate the impact of both the rapid intensification and the crossing of advancing and retreating arrivals during wave focusing on the estimation of the time-varying channel impulse response.

\section{The impact of a caustic}

The transient caustic examined in the prior subsection has several characteristics of importance to the performance of acoustic communications algorithms. These include the sudden appearance of the folded wave front, the advancing and retreating wave fronts in the surface scattered arrival after the appearance of the folded wave front, and rapid changes in the amplitude of the arrival. There may also be a rapid phase shift associated with the caustics but the current data does not provide sufficient information to verify this 

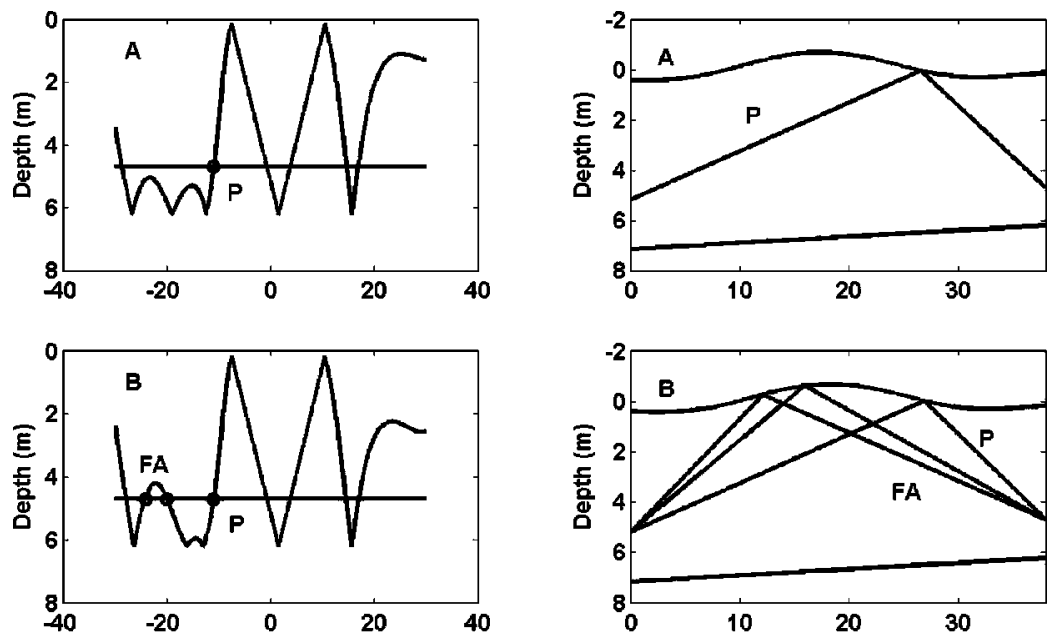

FIG. 9. Modeled angle/depth and ray path diagrams during focusing event. The subfigures labeled A, B, and $\mathrm{C}$ correspond to the three times marked in Fig. 6. The arrival labels (P, FA, FA1, and FA2) correspond to the similarly labeled arrivals in Figs. 6, 7, and 10. The divergence of the saddle points between times $\mathrm{B}$ and $\mathrm{C}$ on the angle/depth diagram results in the formation of two distinct ray paths and arrivals as shown.
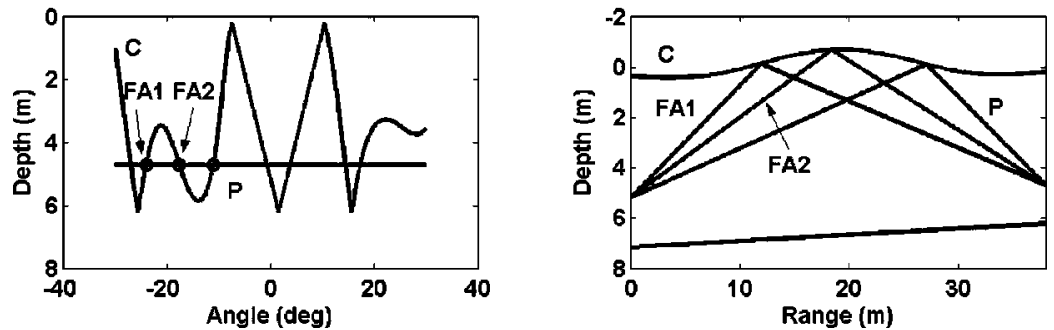

hypothesis. The model results shown in Fig. 6 were generated using an actual surface wave profile from the Wavefronts II experiment. The experimental data corresponding to this wave profile are shown in Fig. 7.

There is a close agreement between the model and experimental results. Comparing the two figures, it is clear that the wave front has been folded back on itself resulting in the formation of caustics and multiple arrivals in the focal region. As was noted previously, there is also some fine structure in the experimental data that is not present in the model results. This is likely due to the limitations of the measurements of the surface wave field used as input data to the Wavefronts model. In addition, as mentioned earlier the finite window averaging used by the least squares channel identification algorithm may result in there being rapidly fluctuating features in the channel impulse response not represented in Fig. 7.

The vertical white lines, labeled $\mathrm{a}-\mathrm{g}$ in Fig. 7 mark significant points of time in the evolution of the surface scattered arrival. The estimated channel scattering function for the surface scattered arrival at each of these points in time is shown in the subfigures of Fig. 10. The scattering function shows the distribution of energy in frequency of the fluctuations of each tap of the channel impulse response (i.e., the horizontal axis) as a function of delay (i.e., the vertical axis). The relative delay axis in the scattering function figures matches the relative delay axis in the impulse response estimate. Energy present at higher frequencies represents arrival energy at a particular delay that is fluctuating more rapidly than energy present at lower frequencies. A description of the scattering function representation of time-varying channels is given in Ref. 8. The distribution of energy in Doppler frequency as a function of relative delay at any of the 8 labeled times in Fig. 7 can be observed by looking at the same rela- tive delay of the corresponding subfigure in Fig. 10.

The movement of energy in the Delay/Doppler plane as the wave crest passes can be explained in terms of the arrivals in Fig. 7 and the model results in Fig. 9. Time a in Fig. 7 corresponds to the shadow zone for the folded wave front. The single ray arrival is the retreating primary arrival (labeled P in Figs. 6-10) and therefore has a negative Doppler shift. This corresponds to time A in Figs. 6 and 9. At time b in Fig. 7, the advancing folded wave front arrival, labeled FA has appeared with a corresponding positive Doppler shift. The arrival has not yet formed a caustic. This feature is not represented in the model results. In the model results, the caustic occurs at the first appearance of the folded wave front arrival. The difference is most likely due to the small scale roughness of the actual sea surface. At time $\mathrm{c}$ in Fig. 7, the caustic is forming as the folded arrival gains in intensity. Accounting for both the primary and folded wave front arrivals, the Doppler spread of this surface scattered arrival is approximately $30 \mathrm{~Hz}$. This time corresponds to time B in Figs. 6 and 9.

Time d in Figs. 7 and 10 corresponds to time C in Figs. 6 and 9. At this time, the caustic has passed, the saddle points shown in Fig. 9 are diverging, and the folded wave front arrival has split into two arrivals labeled FA1 and FA2. The later of the two arrivals (FA2) is scattering off of the peak of the wave crest and is therefore stationary in delay and exhibits no Doppler shift. The earlier of the two arrivals (FA1) is scattering off of the trailing edge of the wave crest and continues to be advancing in delay and exhibit a positive Doppler shift. The evolution of the wave front focusing continues through times $\mathrm{e}-\mathrm{g}$. The arrival FA1 becomes the primary arrival and is advancing. The arrivals FA2 and P eventually merge to form a caustic at time $g$ near the end of the focusing event. 

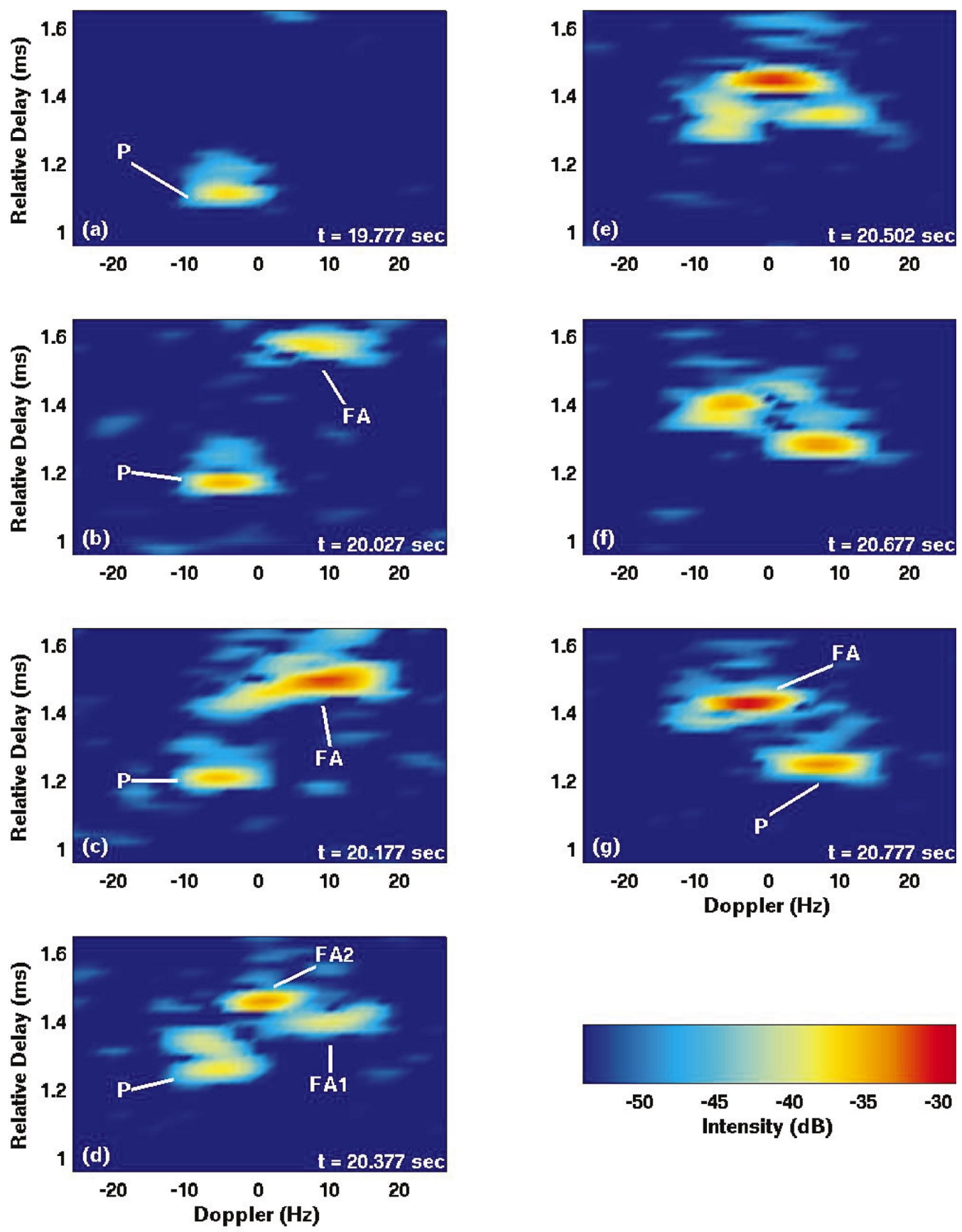

FIG. 10. Estimated channel scattering functions during focusing event. The subfigure labels (a-g) correspond to the similarly labeled points in time in Fig. 7. The arrival labels (P, FA, FA1, and FA2) also correspond to the similarly labeled arrivals in Figs. 6, 7, and 9. The initial primary arrival P is scattering off of the retreating edge of the wave crest and has a negative Doppler shift. The initial folded arrival is scattering off of the advancing edge of the wave crest and has a positive Doppler shift. When the folded arrival splits into two arrivals, the arrival FA2 is scattering off of the peak of the wave crest and has no Doppler shift. FA2 and the initial P arrivals merge to form the final focused arrival scattering off of the retreating edge of the wave crest (FA in subfigure g) with a negative Doppler shift. FA1 becomes the final primary arrival ( $\mathrm{P}$ in subfigure g) which is scattering off of the advancing edge of the wave crest and has a positive Doppler shift. The scattering function estimates were generated with a matched filtering algorithm described in the Appendix and have a frequency resolution of approximately $6.4 \mathrm{~Hz}$.

The simple model of a flat sea surface moving up and down in elevation as used in Ref. 9, and references therein is not sufficient to explain the rate of path length fluctuation for the first surface scattered arrival observed in Fig. 7 and the resulting Doppler spreads observed in Fig. 10. Using the maximum value for $d \eta / d t$ observed during the passage of the wave, the maximum rate of path length change for this arrival is predicted by the simple model to be $0.23 \mathrm{~m} / \mathrm{s}$. The maximum rate of path length fluctuation calculated from the data shown in Fig. 7 is approximately $0.75 \mathrm{~m} / \mathrm{s}$ (arrival FA at time $\mathrm{c}$ in Fig. 7). This difference by over a factor of 3 is due to the effect of the scattering of the focused arrivals off of the advancing and retreating surface wave fronts that propagate at the wave speed (the surface wave speeds observed during the experiment were approximately the $7.5 \mathrm{~m} / \mathrm{s}$ ) and the movement of the scattering point for each arrival along the surface of the wave crest. In addition to the increase in Doppler shift magnitude, focusing from wave crests results in 


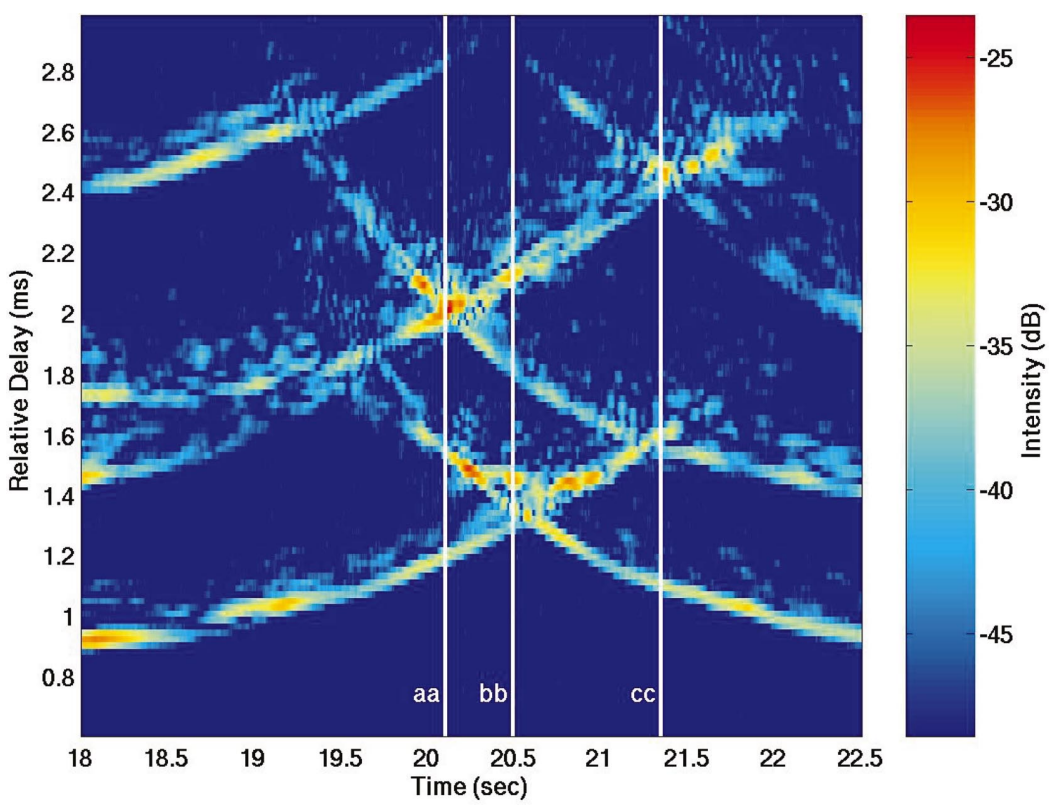

FIG. 11. Three surface scattered arrivals of estimated time-varying channel impulse response during the focusing event. The center of the focusing events for the surface-bottom, surface, and bottom-surface arrivals are labeled aa, bb, and cc, respectively. The sharp peaks in the SER shown in Fig. 12 are labeled in the same manner.

micropath arrivals which exhibit Doppler shifts of opposing signs corresponding to reflections from surface regions on either side of the wave crest maximum. This aspect of the phenomenon would be impossible to reproduce by replacing the wave crest with a moving horizontal surface and presents a problem for commonly implemented phase coherent equalizers.

The impact of the focusing event on the performance of a channel estimation algorithm is illustrated by Figs. 11 and 12. The estimated impulse response in an expanded region in both time and delay around the focusing event is shown in Fig. 11. The focusing event at the time labeled bb is the

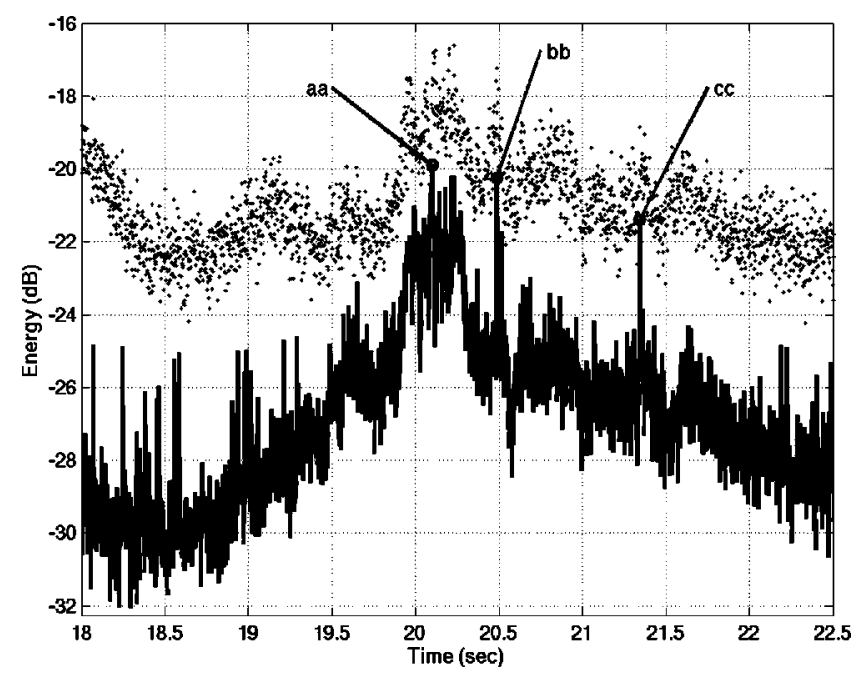

FIG. 12. Signal estimation residual error (SER) and received signal energy during the focusing event. The dots represent received signal energy and the line represents the SER of the least squares channel estimation algorithm. Both quantities are averaged over $1 \mathrm{~ms}$ intervals. The sharp peaks in SER labeled aa, bb, and cc correspond in time with the times at the center of the focusing events for the surface-bottom, surface, and bottom-surface arrivals, respectively, as shown in Fig. 11. surface arrival focus shown in Fig. 7. The arrival whose focusing event occurs at aa is the surface-bottom arrival while the arrival whose focusing event occurs at $\mathrm{cc}$ is the bottomsurface arrival. The received baseband signal energy and the SER of the channel estimation algorithm are shown in Fig. 12. Note that before the passage of the wave crest, the mean error falls between 8 and $10 \mathrm{~dB}$ below the signal energy. As the wave crest passes, the error climbs to within approximately $3 \mathrm{~dB}$ of the received signal energy. This increase in error is a direct result of the increase in the time variation of the channel impulse response as described by the Doppler spreads observed in Fig. 10. Note also the sharp spikes on the signal estimation error with the passage of the center of each focusing event (labeled aa, bb, and cc in Figs. 11 and 12). At these times, the received signal estimation errors are within 1-2 $\mathrm{dB}$ of the received signal levels. This indicates that the channel is highly dynamic at these times and there may be features of the channel impulse response that are not accurately captured in the channel estimates shown in Figs. 7 and 11 . The resolution of these features will be addressed in future work.

A number of the observed features of acoustic focusing events will have detrimental impacts on many current phase coherent demodulation algorithms for underwater acoustic communications. Both channel estimate based algorithms [e.g., channel estimate based decision feedback equalizer (CE-DFE), maximum likelihood sequence estimator] and direct adaptation equalizers (e.g., direct adaptation decision feedback equalizer, DFE) must be able to either explicitly or implicitly track the dominant energy in the time-varying channel impulse response. While in other environments the rapidly varying arrivals may have suffered enough scattering losses to be insignificant, the results here show that the contrary is true in the presence of focusing by surface waves. The focused arrivals have high intensities, often exceeding 
the intensity of the direct arrival, and at the same time are rapidly varying. Therefore, communications algorithms in the surf zone must be able to track these arrivals.

A number of innovations have been made to enable algorithms to track channel fluctuations or reduce algorithm complexity. However, these innovations are based upon a number of simplifying assumptions and they work well as long as the assumptions are satisfied. The features of acoustic focusing events observed here violate most of the important assumptions. For example, the Phase Locked Loop/Signal Resampling approach to addressing path length fluctuations $^{10,11}$ that has been highly successful in other environments is based upon the assumption that a single timevarying Doppler shift/range rate adequately describes the time variation of the channel impulse response. This assumption is clearly violated by both the surface scattered arrival which is significantly Doppler spread and the entire impulse response that consists of fairly stable direct and bottom arrivals in addition to all of the time-varying surface interacting arrivals. In fact, a DFE based upon this approach has not been successful in demodulating the data collected during the focusing event analyzed here.

Another approach to tracking the time-varying channel impulse response is to estimate the channel scattering function periodically during the reception of communications signals and then to track the impulse response for only those points in the Delay/Doppler plane that have significant energy. ${ }^{12,13}$ This technique relies on the assumption that the distribution of energy in the Delay/Doppler plane is stable for periods long enough to allow for initialization and operation of a channel tracking algorithm and the transmission of a packet of data. The evolution of the scattering functions shown in Fig. 10 clearly violates this assumption and would result in a failure of this algorithm.

Finally, channel sparsing algorithms are used to reduce the number of taps of the channel impulse response that are tracked. This sparsing increases the rate of channel fluctuations that can be tracked as well as reduces the computationally complexity of the algorithms. ${ }^{14,15}$ Most sparsing techniques assume that the distribution of energy in the delay variable of the impulse response is relatively stable, i.e., the location of the "significant" taps in the channel impulse reponse is slowly varying. The data shown in Figs. 4, 7, and 11 shows clearly that this assumption is violated for the environmental conditions under which this data was collected. Again, this would result in the failure of these algorithms. Some techniques ${ }^{14}$ do not make this assumption and use other techniques to facilitate tracking of rapid channel fluctuations. However, even these techniques have not been successful in demodulating the data analyzed here.

The results here show that the phase coherent underwater acoustic communications techniques that rely on accurate channel estimation would not be capable of reliable operation in the surf zone environment in the conditions analyzed. This illustrates the need for future work on either improving the ability of channel estimation algorithms to track the fluctuations associated with focusing by surface waves or developing demodulation algorithms that are robust with respect to errors in channel estimates.

\section{STATISTICAL CHARACTERIZATION OF SURFACE WAVE FOCUSING}

The m-seq transmissions made during the Wavefronts II experiment occurred in three $10 \mathrm{~min}$ intervals spread over 2 h. This section presents some sample statistics gathered over these three $10 \mathrm{~min}$ periods. These statistics are calculated from the estimated channel impulse response at $5 \mathrm{~ms}$ intervals, the signal estimation residual error (SER) of the least squares channel estimation algorithm (see Appendix), and the surface wavefield as measured by the array of pressure sensors (see Sec. II).

The measures of SER and surface scattered arrival intensity were examined. These two measures are important for communications applications. Arrival intensity is important because high intensity arrivals present both an opportunity and challenge. Arrivals with high intensity result in a higher signal to noise ratio in the received signal which improves the potential for improved estimation of the transmitted data by the receiver. However, in order to realize this improved data estimation performance, phase-coherent demodulation algorithms must be able to accurately track the phase and amplitude of the arrival. SER is a measure of the ability to do this. When the arrival can be accurately tracked (low SER), the data demodulation algorithm will be able to exploit the higher received signal energy to improve the estimation of the received data. Otherwise (high SER), the received energy represents unmodeled signal and will appear to the receiver to be contaminating noise.

Some of the statistics below were calculated for the entire data set and some were calculated for only that portion of the data that was collected within $100 \mathrm{~ms}$ of the passage of a surface wave. This is referred to as a "wave focusing event." The determination of the time of each wave focusing event was made by time-aligning the surface wave height as measured for each passing wave near the specular point for the surface scattered path with the time series of estimates of the channel impulse response. The fluctuations in the channel impulse response corresponding to the passage of the wave were identified and the center of the focusing event determined. Statistics regarding the behavior of the channel impulse response and the channel estimator were gathered from data within $100 \mathrm{~ms}$ of this center.

Each focusing event was also classified regarding whether or not it resulted in the formation of a caustic. This was done by examining the estimated channel impulse response for the first three surface reflected paths (surface, surface-bottom, bottom-surface) over the duration of the focusing event. If any of these arrivals showed a butterfly like pattern characteristic of a caustic, then the focusing event was classified as having resulted in the formation of a caustic. Otherwise, it was classified as not having resulted in the formation of a caustic.

The sample pdfs of the log intensity $\left[10 * \log _{10}\right.$ (intensity)] of the estimated direct and first surface bounce path arrivals are shown in Fig. 13. In contrast to the results shown in Fig. 1 which show the maximum and mean intensity for each delay tap in the estimated time-varying impulse response, Fig. 13 shows the intensity statistics for estimated arrivals which, for the case of the surface bounce path ar- 


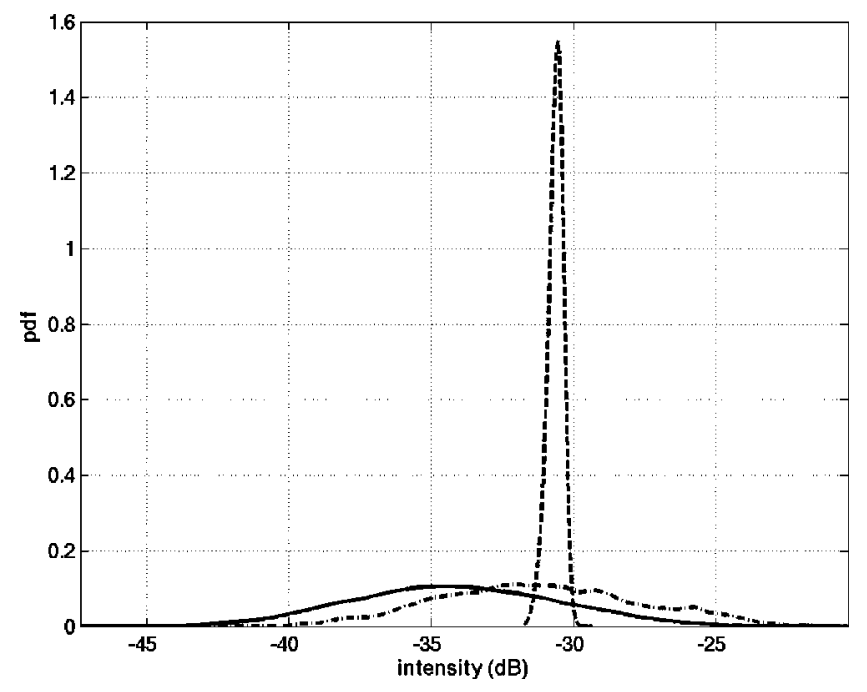

FIG. 13. Sample pdf of $10 * \log _{10}$ of the intensities of estimated direct and surface bounce path arrivals. The dashed line is the pdf for the direct arrival, the solid line is the pdf for the first surface bounce path arrival. The dasheddotted line is also the pdf for the first surface bounce path arrival but using data only from within the interval extending from $100 \mathrm{~ms}$ before to $100 \mathrm{~ms}$ after the time of each wave focusing event.

rival, move in tap position (delay) as the surface elevation fluctuates. The pdf for the surface bounce path arrival is calculated both over the entire data collection period and over the intervals within $100 \mathrm{~ms}$ of each wave focusing event. The mean values for the peak intensity of the estimated direct arrival, the surface path arrival, and the surface path arrival within $100 \mathrm{~ms}$ of each wave focusing event, are $-30.63 \mathrm{~dB},-32.62 \mathrm{~dB}$, and $-29.50 \mathrm{~dB}$, respectively.

The pdf for $10 * \log _{10}$ of the ratio of the peak intensity of the estimated surface bounce path to the estimated peak intensity of the direct path for each estimated channel impulse response is shown in Fig. 14. The pdfs were calculated both over the entire data set and over only the data taken within $100 \mathrm{~ms}$ of each wave focusing event. For the full data set, the intensity of the estimated surface bounce path exceeds that of the direct path $17.7 \%$ of the time. For the data falling within $100 \mathrm{~ms}$ of each wave focusing event, the intensity of the estimated surface bounce path exceeds that of the direct path $45.7 \%$ of the time.

The impact of surface wave focusing on the intensity of the surface scattered arrival are clearly illustrated by Figs. 13 and 14. Separate intensity statistics were also calculated for focusing events that showed the formation of a caustic and those that did not. The intensity pdfs for these two classes of focusing events were virtually identical. This indicates that, to the extent that the channel fluctuations are accurately tracked by the channel estimation algorithm, the intensity statistics during the passage of a wave do not depend on whether or not a caustic is formed.

The mean of the SER conditioned upon the delay of the first surface bounce path arrival is shown in Fig. 15. The data clearly shows that the mean SER increases as the delay increases. Since the delay of this arrival is maximum at or near the focusing event, this shows that the SER is generally maximized at or near the focusing events. This conditional mean was calculated from using the entire data set.

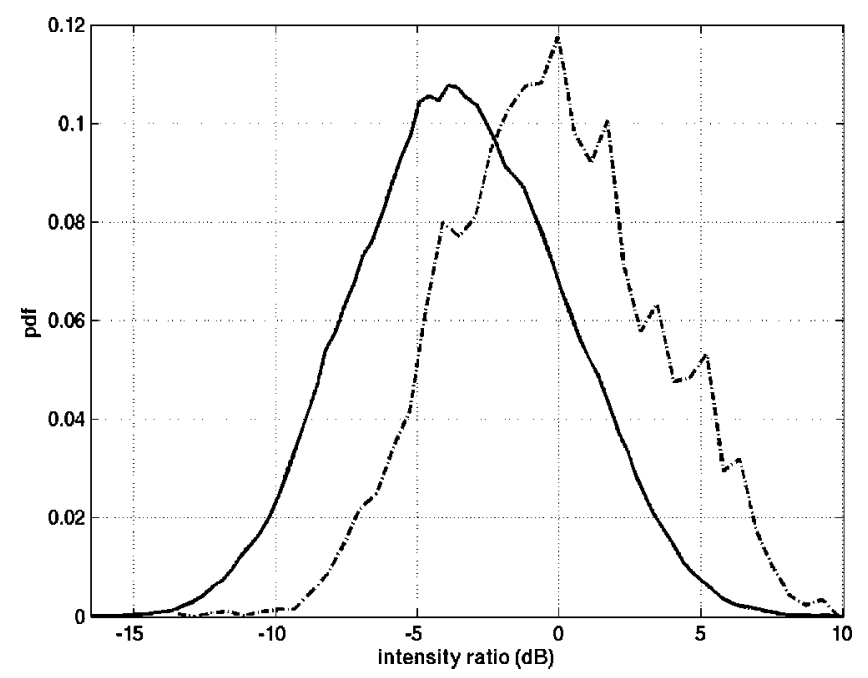

FIG. 14. Sample pdf of $10 * \log _{10}$ of the ratio of the intensities of the direct path to surface bounce path arrivals. The $x$-axis scale indicates the amount by which the intensity of the first surface bounce path arrival exceeded that of the direct path arrival. The solid line shows the pdf calculated using the entire $30 \mathrm{~min}$ data set. The dashed-dotted line shows the pdf calculated using data only from within the interval extending from $100 \mathrm{~ms}$ before to $100 \mathrm{~ms}$ after the time of each wave focusing event.

Scatter plots of the mean SER taken over intervals extending from $100 \mathrm{~ms}$ before to $100 \mathrm{~ms}$ after each focusing event are shown in Fig. 16. There were some focusing events in the data for which the data are not included in Fig. 16. These data were from events involving waves for which multiple wave peaks overlapped or other features made it difficult to calculate either a wave height or focal length for the portion of the wave that resulted in the focusing. The data for the events that formed caustics shows a clear trend of increasing SER with both a decreasing focal length and increasing wave height. The data for events that did not form caustics shows only a weak dependence of SER on focal length but a clear trend of increasing SER with increasing wave height. This is in contrast to the lack of a dependence

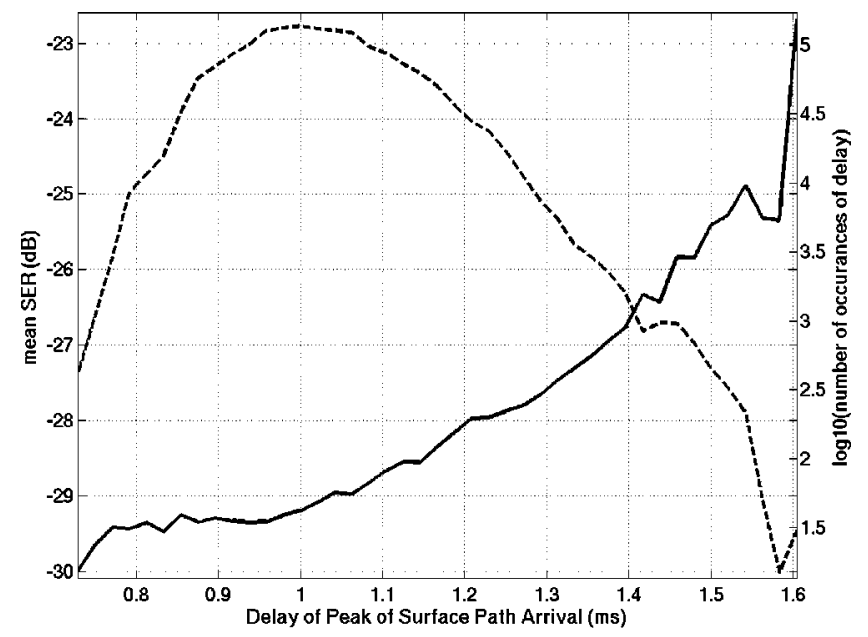

FIG. 15. Sample mean SER conditioned on the delay of the peak of the first surface bounce path arrival. The solid line (scale on left vertical axis) is the mean SER and the dashed line (scale on right vertical axis) represents the number of occurrences in logarithmic units of the peak of the first surface bounce path arrival being located at the indicated delay. 

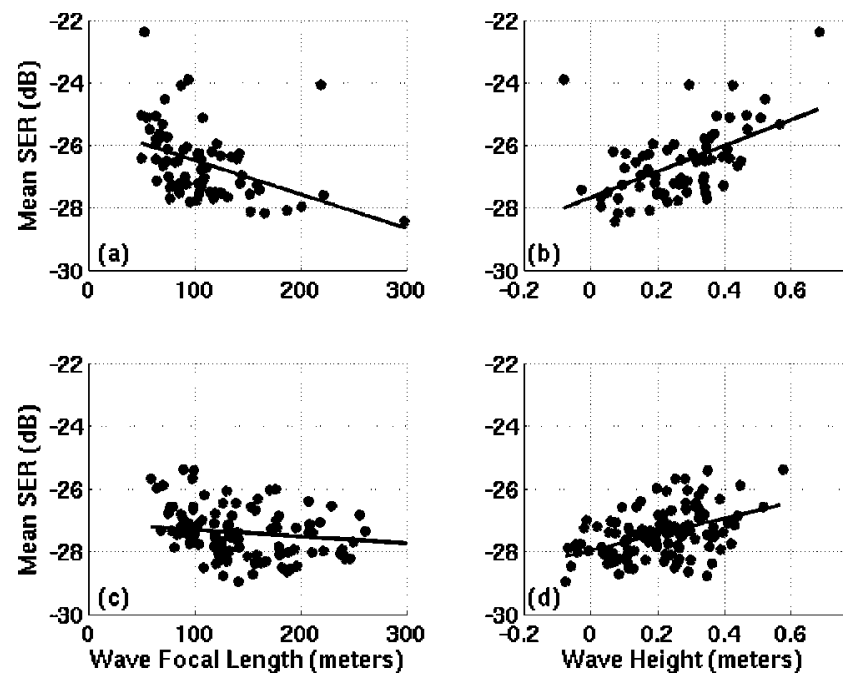

FIG. 16. Sample mean SER during the passage of each surface wave as a function of wave focal length and wave height. The surface wave events are divided into those which produced a clear caustic focusing event [(a) and (b)] and those which did not [(c) and (d)]. The line in each plot is a linear fit to the data in each plot. The mean for each wave was taken over an interval extending from $100 \mathrm{~ms}$ before to $100 \mathrm{~ms}$ after the time of each wave focusing event. For comparison, the mean SER over the entire $30 \mathrm{~min}$ data set is $-28.61 \mathrm{~dB}$.

of arrival intensity statistics on whether or not a caustic is formed as discussed earlier.

The total number of events recorded was 185 . Of these, 123 events had surface wave focal lengths of greater than $100 \mathrm{~m}$. Thirty five $(28 \%)$ of these events showed the formation of a caustic while $88(72 \%)$ did not. Sixty two events had surface wave focal lengths of less than $100 \mathrm{~m}$. Thirty four $(55 \%)$ of these events showed the formation of a caustic while $28(45 \%)$ did not. These statistics show that the likelihood of the formation of a caustic increases for surface waves with focal lengths approaching the propagation path length of approximately $40 \mathrm{~m}$. Of the 34 events with focal lengths less than $100 \mathrm{~m}$ and that showed the formation of a caustic, $13(38 \%)$ resulted in a SER of greater than -25.76 $\mathrm{dB}$. Of the 28 events with focal lengths less than $100 \mathrm{~m}$ and that did not show the formation of a caustic, only 4 (14\%) resulted in a SER of greater than $-25.76 \mathrm{~dB}$. Thus, the increased likelihood of the formation of a caustic by short focal length waves also results in a generally increased SER indicating poor tracking of the channel impulse response and an inability to exploit the increased surface arrival energy.

The data shows that the SER statistics depend on whether or not a caustic is formed but the arrival intensity statistics do not. This difference may be the result of several factors. First, the result may accurately represent the propagation physics. Second, the small scale surface roughness that is not measured by the pressure sensor array (see Sec. II) may be an important element in controlling the intensity fluctuations of the surface scattered arrival. That is, there may be surface waves that have scales large enough to focus sound with an $8.3 \mathrm{~cm}$ wavelength but which are too small to be accurately measured by bottom mounted pressure sensors in approximately $6 \mathrm{~m}$ of water. These waves may also have surface normal velocities that are too small to result in significant Doppler shifts in the surface scattered arrivals. If this were the case, these small scale waves may significantly impact the surface scattered arrival intensity statistics but not the SER statistics and would not be reflected in the measured surface wave data. Finally, the higher SER during focusing events in which a caustic is formed may indicate that there are higher intensity arrivals during these events that the channel estimation algorithm is unable to track. Hence, these higher intensity arrivals would not be reflected in the intensity statistics presented here. The currently available data is not sufficient to resolve this issue.

\section{CONCLUSIONS}

The acoustic focusing caused by the scattering of signals off of surface gravity waves in the surf zone gives rise to arrivals that have both high intensity and are rapidly fluctuating. In the experimental data presented, the intensity of these arrivals at times exceed that of the direct arrival by almost $10 \mathrm{~dB}$. During the peak of the passage of a surface wave, the simultaneous presence of advancing and retreating wave-fronts in the surface scattered arrival results in a Doppler spread of the arrival by up to $30 \mathrm{~Hz}$. The high intensity and rapid fluctuation of the surface scattered arrival during a focusing event significantly degrades the performance of a least squares algorithm for estimating the channel impulse response. This will significantly degrade the performance of phase coherent acoustic communications systems that must either implicitly or explicitly estimate the channel impulse response.

The evaluation of surface wave and acoustic data from a single focusing event shows close agreement between model and experimental results. The model data clearly shows the important features in the focusing event including the formation of caustics. The experimental data clearly shows a peak in signal estimation residual error (SER) at a particular phase of the focusing event. However, the currently available data is not sufficient to resolve the precise features of the channel impulse response that results in an error spike at these times. The evaluation of data from 185 passing surface waves over 30 min of data collection showed that for those 69 focusing events that showed the formation of a caustic, the SER showed a strong increase with the decreasing focal length of the wave and with the increasing height of the wave. For those events that did not show the formation of a caustic, the SER showed little dependence on the focal length of the wave but did show a increase with increasing wave height.

The overall conclusion that can be drawn is that surface wave focusing causes an increase in the amplitude of the surface scattered arrivals at a receiver and a degradation in the ability of an estimation algorithm to track the fluctuations in the channel impulse response. For events involving the formation of a caustic at the receiver, the degradation can be related to the wave parameters of focal length and wave height. Additional work is needed to further resolve the characteristics of the fluctuations in the channel impulse response in the vicinity of the caustic and the primary cause of the degradation in algorithm performance. Two methods of resolving these issues would be to conduct tests with shoaling surface waves propagating in a well controlled tank environment and to conduct tests using the transmission of short 
acoustic pulses with a level sufficient to yield reliable estimates of the channel impulse response without the need for temporal averaging.

\section{ACKNOWLEDGMENTS}

The authors are pleased to acknowledge Dr. Chris Tindle for many insightful discussions and creating a version of his Wavefronts model suitable for use in the surf zone. We would also like to acknowledge Dr. Dale Stokes for his assistance with the field work. This work has been supported by ONR Grant Nos. N00014-96-1-0120, N00014-00-1-0303, N00014-99-1-0274, and N00014-00-1-0048. This paper is WHOI Contribution No. 11018.

\section{APPENDIX: CHANNEL IMPULSE RESPONSE AND SCATTERING FUNCTION ESTIMATION}

The acoustic signals received from each of the experiments were processed to yield estimates of the time-varying impulse response of the acoustic channel. The received signals for the maximum length shift register sequence (m-seq) transmissions (see Sec. II) were modulated to baseband, lowpass filtered, and then downsampled to a rate of two samples per symbol. The resulting baseband signals were then used in combination with the original data sequence as the inputs to a least squares channel identification algorithm to generate estimates of the baseband channel impulse response. The duration of the modulation pulse used in the transmitted signals was approximately $0.04 \mathrm{~ms}$. The impulse response estimates derived from the data are actually estimates of the channel impulse response convolved with this modulation pulse and have resolution in delay of approximately $0.04 \mathrm{~ms}$.

The particular form of the baseband channel impulse response estimated by this method is the input delay-spread function. ${ }^{8}$ The channel input/output relationship for the sampled input delay-spread function is $y[n]$ $=\sum_{m=0}^{N_{c}} g *[n, m] d[n-m]$, where $g[n, m]$ denotes the baseband input delay-spread function as a function of output sample index, $n$, and sample delay, $m, N_{c}$ is the number of causal samples in the delay dimension of the input delayspread function $\left(N_{c}=167\right.$ was used), the superscript $*$ denotes complex conjugate, $d[n]$ is the baseband transmitted data sequence, and $y[n]$ is the sampled baseband output of the channel. Letting

$$
\mathbf{g}[n]=\left[\begin{array}{c}
g[n, 0] \\
\vdots \\
g\left[n, N_{c}\right]
\end{array}\right], \quad \mathbf{d}[n]=\left[\begin{array}{c}
d[n] \\
\vdots \\
d\left[n-N_{c}\right]
\end{array}\right],
$$

and $v[n]$ denote the baseband observation noise, the baseband received signal $y[n]$ is given by $y[n]=\mathbf{g}[n]^{h} \mathbf{d}[n]$ $+v[n]$. Here, the superscript $h$ denotes Hermitian and boldface letters denote vectors.

The least squares algorithm used a sliding, rectangular data window to accommodate time variation in the channel impulse response. The delay spread of the estimated channel impulse response was $7 \mathrm{~ms}$ (168 symbol periods) and the rectangular averaging window was $25 \mathrm{~ms}$ (600 symbol periods) in length. The algorithm was run with averaging windows ranging from 10 to $45 \mathrm{~ms}$ and the best results in terms of minimizing the signal estimation residual error were achieved using the $25 \mathrm{~ms}$ averaging window. At each symbol period, the estimated impulse response vector is given by $\hat{\mathbf{g}}[n]=\arg \min _{g} \sum_{k=0}^{599}\left|y[n-k]-\mathbf{g}^{h} \mathbf{d}[n-k]\right|^{2}$. This estimated impulse response was saved every $5 \mathrm{~ms}$ (120 symbol periods). The fractional spacing of the received baseband signal at 2 samples per symbol was accommodated by running 2 independent channel identification algorithms (one for odd numbered samples and the other for even number samples) and interleaving the resulting channel impulse response estimates.

The signal estimation residual error (SER) is given by $e[n]=y[n]-\hat{\mathbf{g}}[n-1]^{h} \mathbf{d}[n]$ and is used as a measure of how well the estimated channel impulse response approximates the actual channel impulse response. That the energy in $e[n]$ is a reasonable measure of the agreement between the estimated and actual channel impulse response is justified in the following paragraph.

The estimate of the impulse response at time $n$ using received signal data collected up to time $(n-1)$ is denoted by $\hat{\mathbf{g}}[n-1]$. Then the residual prediction error can be expressed as

$$
\begin{aligned}
e[n] & =y[n]-\hat{\mathbf{g}}[n-1]^{h} \mathbf{d}[n] \\
& =(\mathbf{g}[n]-\hat{\mathbf{g}}[n-1])^{h} \mathbf{d}[n]+v[n] .
\end{aligned}
$$

Assuming that the baseband observation noise and data sequence are zero-mean and uncorrelated, the baseband data sequence has a variance of one and is a white sequence, the variance of the baseband observation noise is $\sigma_{v}^{2}$, and that the impulse response is uncorrelated with both the baseband observation noise and data sequence, the variance of the residual prediction error is give by

$$
E \mid[e[n]]=\sigma_{e}^{2}[n]=\|\mathbf{g}[n]-\hat{\mathbf{g}}[n-1]\|^{2}+\sigma_{v}^{2} .
$$

Here the expectation is with respect to the observation noise and baseband transmitted data sequence. Thus, with the assumption that the variance of the observation noise is constant, $\sigma_{v}^{2}$, the signal estimation residual error is a reasonable measure of the 2-norm of the difference between the actual and estimated channel impulse response.

The channel scattering function ${ }^{8}$ was estimated by matched filtering frequency shifted versions of the received baseband signal with a windowed 4095 point m-seq. The window was used to reduce sidelobe levels at the expense of mainlobe width. The window used was a Kaiser window with a shape parameter of 3 . This yielded a maximum sidelobe level of $-23.8 \mathrm{~dB}$ relative to the peak mainlobe level and a two-sided mainlobe $3 \mathrm{~dB}$ width of $6.4 \mathrm{~Hz}$.

\footnotetext{
${ }^{1}$ J. A. Smith, "Performance of a horizontally scanning Doppler sonar near shore," J. Atmos. Ocean. Technol. 10, 752-763 (1999).

${ }^{2}$ D. M. Farmer, G. B. Deane, and S. Vagle, "The influence of bubble clouds on acoustic propagation in the surf zone," IEEE J. Ocean. Eng. 26, 113124 (2001).

${ }^{3}$ K. L. Williams, J. S. Stroud, and P. L. Marston, "High-frequency forward scattering from Gaussian spectrum, pressure release, corrugated surfaces. I. Catastrophe theory modeling," J. Acoust. Soc. Am. 96, 1687-1702 (1994).

${ }^{4} \mathrm{C}$. T. Tindle, "Wavefronts and waveforms in deep-water sound propagation," J. Acoust. Soc. Am. 112, 464-475 (2002).
}

${ }^{5}$ M. J. Buckingham, "Precision correlations between the geoacoustic pa- 
rameters of an unconsolidated, sandy marine sediment," J. Comput. Acoust. 9, 101-123 (2001)

${ }^{6}$ J. G. Proakis, Digital Communications, 3rd ed. (McGraw-Hill, New York, 1995), Chap. 13, pp. 724-729.

${ }^{7} \mathrm{G}$. B. Deane, "The beam forming properties of a concave spherical reflector with an on-axis receiver," J. Acoust. Soc. Am. 106, 1255-1261 (1999).

${ }^{8}$ P. A. Bello, "Characterization of randomly time-variant linear channels," IEEE Trans. Commun. Electron. CS-11, 360-393 (1963).

${ }^{9}$ M. Stojanovic, "Recent advances in high-speed underwater acoustic communications," IEEE J. Ocean. Eng. 21, 125-136 (1996).

${ }^{10}$ M. Stojanovic, J. Catipovic, and J. Proakis, "Adaptive multichannel combining and equalization for underwater acoustic communications," J. Acoust. Soc. Am. 94, 1621-1631 (1993).

${ }^{11}$ M. Johnson, L. Freitag, and M. Stojanovic, "Improved Doppler tracking and correction for underwater acoustic communications," Proc. ICAASP'97, Munich, Germany, 1997, pp. 575-578.

${ }^{12}$ T. Eggen, A. Baggeroer, and J. Preisig, "Communication over Doppler spread channels-Part I: Channel and receiver presentation," IEEE J. Ocean. Eng. 25, 62-71 (2000).

${ }^{13}$ T. Eggen, J. Preisig, and A. Baggeroer, "Communication over Doppler spread channels-Part II: Receiver characterization and practical results," IEEE J. Ocean. Eng. 26, 612-621 (2001).

${ }^{14}$ M. Stojanovic, L. Freitag, and M. Johnson, "Channel-estimation-based adaptive equalization of underwater acoustic signals," OCEANS'99 Conference, Seattle, WA, 1999, pp. 985-990.

${ }^{15}$ M. Stojanovic, "Efficient acoustic signal processing based on channel estimation for high rate underwater information," J. Acoust. Soc. Am. (submitted). 